\title{
Reproducibility of straylight measurement by C-Quant for assessment of retinal straylight using the compensation comparison method
}

\author{
Ivo Guber • Lucas M. Bachmann • Josef Guber • \\ Frank Bochmann • Alex P. Lange • Michael A. Thiel
}

Received: 13 December 2010 /Revised: 29 March 2011 /Accepted: 30 March 2011 / Published online: 13 May 2011

(C) Springer-Verlag 2011

\begin{abstract}
Background Straylight gives the appearance of a veil of light thrown over a person's retinal image when there is a strong light source present. We examined the reproducibility of the measurements by C-Quant, and assessed its correlation to characteristics of the eye and subjects' age. Participants and Methods Five repeated straylight measurements were taken using the dominant eye of 45 healthy subjects (age 21-59) with a BCVA of 20/20: 14 emmetropic, 16 myopic, eight hyperopic and seven with astigmatism. We assessed the extent of reproducibility of straylight measures using the intraclass correlation coefficient.

Results The mean straylight value of all measurements was 1.01 (SD 0.23, median 0.97, interquartile range 0.85-1.1).

Per 10 years of age, straylight increased in average by 0.10
\end{abstract}

Presented at the 102nd Annual Meeting of the Swiss Society of Ophthalmology (SSO), Lugano, Switzerland, September 2nd to 5th, 2009

Presented at the ARVO 2010 Annual Meeting, Fort Lauderdale, Florida, USA, May 2-6 2010

I. Guber $(\bowtie)$

Jules-Gonin Eye Hospital, University of Lausanne,

Avenue de France 15,

1000 Lausanne 7, Vaud, Switzerland

e-mail: ivo.guber@fa2.ch

I. Guber $\cdot$ J. Guber $\cdot$ F. Bochmann $\cdot$ M. A. Thiel

Eye Clinic, Cantonal Hospital of Lucerne,

Lucerne, Switzerland

L. M. Bachmann

medignition Inc. Research Consultants,

Zug, Switzerland

A. P. Lange

Department of Ophthalmology, Toronto Western Hospital,

Toronto, Ontario, Canada
(95\% CI 0.04 to $0.16, p<0.01]$. We found no independent association of refraction (range $-5.25 \mathrm{dpt}$ to $+2 \mathrm{dpt}$ ) on straylight values $(0.001 ; 95 \% \mathrm{CI}-0.022$ to $0.024, p=0.92)$. Compared to emmetropic subjects, myopia reduced straylight $(-.011 ;-0.024$ to $0.02, p=0.11)$, whereas higher straylight values $(0.09 ;-0.01$ to $0.20, p=0.09)$ were observed in subjects with blue irises as compared to darkcolored irises when correcting for age. The intraclass correlation coefficient (ICC) of repeated measurements was 0.83 ( $95 \%$ CI 0.76 to 0.90$)$.

Conclusions Our study showed that straylight measurements with the C-Quant had a high reproducibility, i.e. a lack of large intra-observer variability, making it appropriate to be applied in long-term follow-up studies assessing the long-term effect of surgical procedures on the quality of vision.

Keywords C-Quant · Scatter · Straylight · Compensation comparison method

\section{Introduction}

While visual acuity has traditionally been seen as the cornerstone of ophthalmologic care and visual function, the role of glare and contrast sensitivity has recently gained importance when assessing the quality of vision [1]. Consideration of these additional parameters has become particularly important in cataract and refractive surgery, where restoration of excellent visual acuity may not necessarily lead to complete patient satisfaction if the vision is tinged by troublesome glare [1].

Straylight is the known cause of disability glare [2-4]. Light scattering in the eye's optical media causes a veil of straylight over the retina. This leads to deleterious visual effects such as glare while driving at night, hindrance from 
a low sun during daytime, facial recognition problems, reports of haziness of vision, color and contrast loss [2-4]. Because typical straylight-dependent symptoms occur independently from visual acuity-associated symptoms [5], the assessment of straylight would be an important element in the clinical work-up if measurements are reproducible.

The straylight meter (Oculus Optikgeräte, GmbH, Wetzlar, Germany) directs a forward scatter light into the eye, and allows the resultant scattered light to be compared with a reference light. The patient controls the reference light, which is matched to the scattered light, thus allowing the amount the eye scatters light to be quantified [3]. In 1992, Beckman and colleagues found a correlation between the extent of patients' glare assessed with a questionnaire and the extent of straylight [5]. Since 2005 a computerized straylight meter, called the C-Quant (Oculus Optikgeräte, $\mathrm{GmbH}$, Wetzlar, Germany) has been commercially available. The new device works with a psychophysical method, the so-called compensation comparison [6,7]. A first publication on 20 young volunteers reported excellent validity and reliability in clinical tests, as well as resistance to fraud [8]. Another study found advantages compared to the non-computerized straylight meter [9].

Although this new straylight meter may be superior to other glare tests, clinical experience is yet limited. Quantifying straylight could provide valuable insights into visual function, but reproducibility of this measurement has not been fully assessed and the knowledge of factors influencing straylight is still limited.

In this study, we built on previous research, and aimed at examining the reproducibility of the measurements and assessed the association of refraction, eye pigmentation, and subject's age on straylight measurements.

\section{Methods}

This study was approved by the local ethics committee.

\section{Participants}

To test the reproducibility of the clinical version of the compensation comparison method, 45 healthy Caucasian subjects without training in the direct compensation and compensation comparison method were recruited from the hospital staff. All participants gave their informed consent prior to their inclusion in the study. We performed a full eye examination including slit lamp and fundoscopy.

Straylight measurements

Intraocular straylight was quantified using the Oculus C-Quant meter, which works with the compensation comparison method. The method was previously described in detail $[6,7]$. In brief, the meter compares the intensity of a counterphase flickering that is required to compensate an induced flickering that is considered a proxy for intraocular straylight.

All measurements were performed on the subjects' dominant and undilated eye, without glasses or contact lenses, while the other eye was covered. Thoroughly cleaned trial glasses were used if necessary. All subjects performed five repeated measurements after a short instruction. The instruction phase, a feature providing five stimuli to familiarize a subject with the flicker comparison task, and to verify whether the subject is able to perform this task, was always activated at every measurement. The measurements took place in a darkened and quiet room. All measurements started with the default setting (position $\mathrm{E}$ on the device). If the system considered the measurement to be unreliable, the setting was adjusted to the point where reliable measurements could be obtained. Only measurements with an estimated standard deviation (ESD) and quality factor for psychometric sampling (Q) lower than 0.08 or higher than 1.00 as displayed by the C-Quant meter were used for analysis [6].

\section{Statistical analysis}

We summarized interval scaled variates with medians and interquartile ranges (IQR) or means and standard deviations (SD) where appropriate. Dichotomous variates were described as ratios and percentages.

To explore the strength of association of participants' age, state of refraction (emmetropia, astigmatism, hyperopia, and myopia), refraction and iris color (blue or green vs brown) (independent variates) on straylight measurements (dependent variate), we fitted a mixed linear model entering age as a continuous variate, state of refraction using three indicator variates, refraction as a continuous variate, and iris color as a dichotomous variate into the model. To adjust for the fact that measurements were independent between subjects but dependent within subjects, we entered the subject variate as a random factor. The results are presented with the mean coefficient, the $95 \%$ confidence interval $(95 \% \mathrm{CI})$ and the corresponding $p$ value. A $p$-value of less than 5 percent was considered statistically significant.

Finally, we also calculated the intraclass correlation coefficient (ICC) for the straylight measurement using a one-way random effects model. ICC values can range from 0 to 1 , where a value of 1 indicates a complete agreement between the measurements. Based on the paper by Landis and Koch on the interpretation of Kappa coefficients, we considered ICC values of 0.21 to 0.40 to indicate "fair", values of 0.41 to 0.60 to indicate "moderate", values of 
0.61 to 0.80 to indicate "substantial", and values of 0.81 to 1.00 to indicate "excellent" agreement [10].All analyses were performed using the Stata 11 statistics software package (StataCorp LP, 4905 Lakeway Drive, College Station, TX, USA)

\section{Results}

None of the participants showed evidence for ocular defects or had a history of previous ocular surgery. Refraction ranged from $+2.0 \mathrm{D}$ to $-5.25 \mathrm{D}$. All subjects had a BCVA of 20/20, of whom 14 were emmetropic, 16 were myopic, eight were hyperopic and seven had astigmatism (see Table 1)

The average age was 33.13 years (SD 10.25, median 29 years, IQR 26-39 years).

Five repeated measurements were taken per eye with a total of 225 measurements. The mean straylight value of all measurements was 1.01 (SD 0.23, median 0.97, IQR 0.851.1). The mean investigation duration for all measurements was $1.30 \mathrm{~min}$ (SD 0.10, median 1.27, IQR 1.25-1.33). Figure 1 shows an overview of the performed measurements by subject.

Per 10 years of age, straylight increased in average by $0.10(95 \%$ CI 0.04 to $0.16, p<0.01)$. (see Fig. 2) We found no effect of refraction on straylight values. Per dioptre increase straylight only increased minimally $(+0.001 ; 95 \%$ CI -0.022 to $0.024, p=0.92$ ). Compared to emmetropic subjects we observed reduced straylight in myopes(-.011; -0.024 to $0.02, p=0.11)$, and higher values for hyperopes $(0.108 ;-0.130$ to $0.347, p=0.37)$ and astigmates $(0.021$; -0.173 to $0.214, p=0.83)$. In contrast, higher straylight values $0.09(-0.01$ to 0.20$), p=0.09)$ were observed in subjects with blue irises as compared to dark-colored irises when correcting for age and in astigmats $(0.11 ;-0.13$ to $0.35, p=0.37$ ). The Intraclass correlation coefficient (ICC) of repeated measurements was 0.83 (95\%CI 0.76 to 0.90$)$.

Table 1 Population descriptives

\begin{tabular}{ll}
\hline Subjects, $n$ & 45 \\
Male/female & $10 / 35$ \\
Blue or green/dark-colored Irises & $19 / 26$ \\
Age, years, mean (SD) (range) & $33.13,10.34(21$ to 59$)$ \\
SE refraction, $D$, mean, SD (range) & $-1.26,1.80(-5.0$ to 2.0$)$ \\
Emmetropes, $n$ (range) & $14(-0.25$ to +0.25$)$ \\
Myopes, $n$ (range) & $16(-1.25$ to -5.25$)$ \\
Hypertropes, $n$ (range) & $8(+0.75$ to +2$)$ \\
Astigmats, $n$ (range) & $7(+0.75$ to +2.25$)$ \\
\hline
\end{tabular}

\section{Discussion}

We found excellent reproducibility of measurements, and showed that straylight was strongly correlated with increasing age, whereas light irises and type of refraction had only a minor effect. Myopes tended to lower, while hyperopic subjects and subjects with astigmatism tended to higher straylight values. Higher straylight values were also observed in subjects with blue irises as compared to darkcolored irises. However, all these differences did not reach statistical significance.

Cervino et al. examined the straylight values of 20 young patients (mean age 26.9 years) taking ten repeated measures, and found a good reliability [8]. We were able to reproduce this finding, and calculated an Intraclass correlation coefficient of 0.83 , which corresponds to an excellent reproducibility. A recent study by Rozema et al. examined straylight values of 518 subjects as a function of the axial length. They showed that the base-and-age corrected straylight (BAC straylight) increased with increasing axial length, resulting in higher straylight values for myopes [11]. Our study, however, basically found no correlation between refraction and straylight. Moreover, our data also speak against the Rozema finding with respect to refraction, because we observed a tendency for lower values in myopes which was statistically not significant. Arguably, this discrepancy could be explained with differences in study populations. The present study only included healthy subjects with a BCVA of 20/20 and without any additional changes due to the myopia such as retinopathy or posterior staphyloma. The influence of such changes on straylight is unclear, and needs further investigation.

Dry eyes and/or the tear film could be other sources with an effect on straylight. This is particularly interesting, since the assessment itself could trigger changes in tear film. The examination lasts about 90 seconds and every person receives roughly 25 stimuli per measurement. It cannot be completely ruled out that patients get excited or strained. Moreover, the tendency to complete the examination without blinking could lead to a breakup of the tear film and to an irregular corneal surface. In practice however, these effects were not very important. We observed that, if the test persons did not blink regularly, the system did not supply reliable values as indicated by a low quality score.

Because excellent visual acuity is now achievable from cataract and refractive surgery, the drive for measurement of broader aspects of visual function has increased. Some patients who have moderate visual acuity preoperatively may not be prepared to accept good visual acuity postoperatively if it is tinged by troublesome glare or loss of contrast sensitivity. When a patient states that they have problems with glare, there are many distinct visual phenomena that they may be describing. Disability glare 


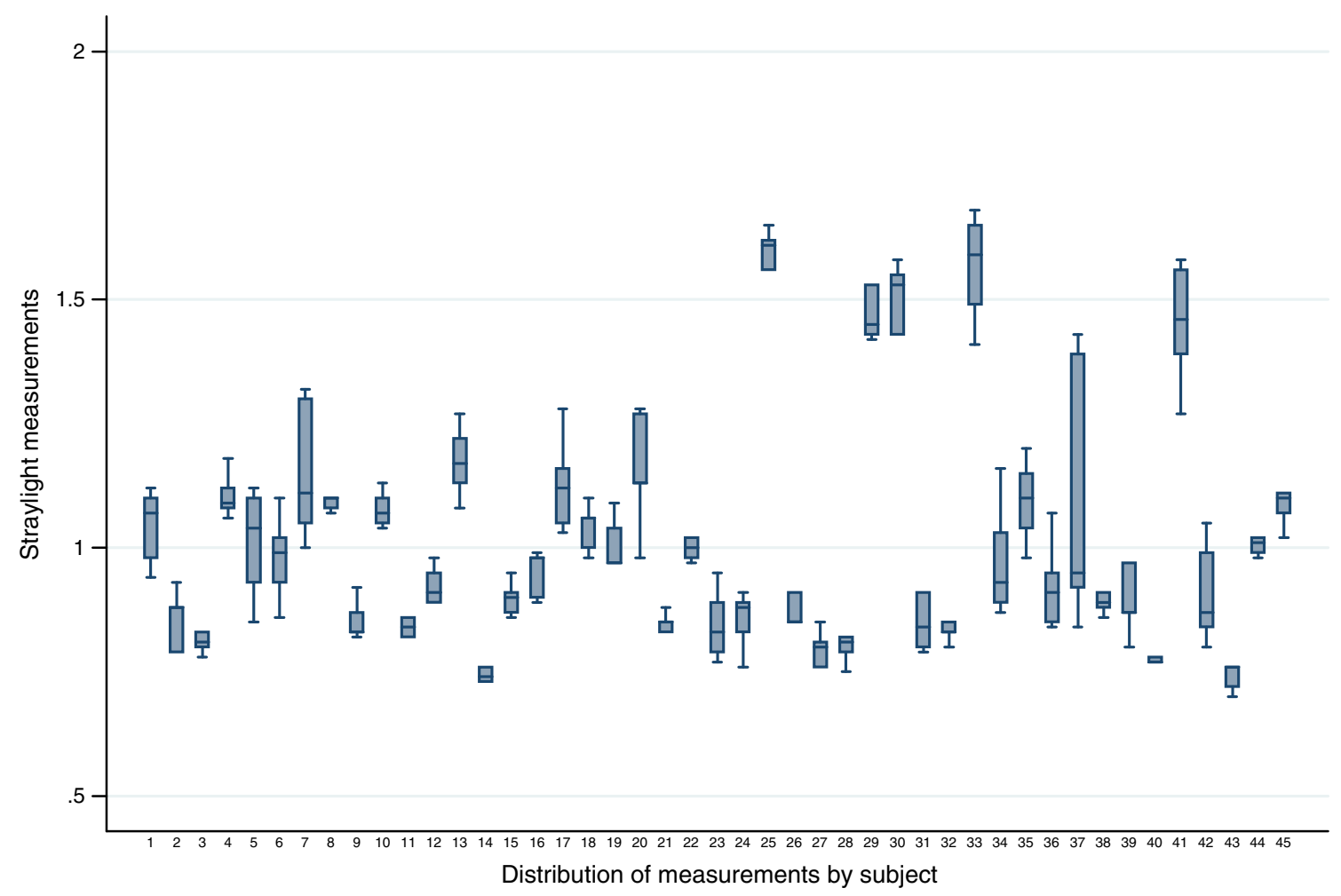

Fig. 1 Distribution of straylight measurements by subject. Footnote: $X$ : Subjects 25, 29 and 33 are between 20 and 30 years of age, with blue or green eyes, whereas subject 30 has the age of 58 years and

is the loss of retinal image contrast as a result of intraocular light scatter, or straylight. It has been described as a reduction of visual acuity caused by light elsewhere in the field of vision [12]. For example, when driving at night, oncoming headlights might reduce visual function [13]. Disability glare gives an understanding of the patient's actual visual impairment. Straylight, and the equations that describe it, give different and more specific information on the quality of the eye's optics [14]. Studies have shown that tests of visual dark eyes. Subject 37 in return complained about dry eyes, had an irregular tear film and showed reduced blinking

acuity measured in the visually impaired do not necessarily predict problems encountered in everyday life, especially in those with mild visual impairment $[15,16]$.

In summary, our study suggests that the C-Quant reproducibly measures straylight. Measurements are only modestly affected by the refractive state of the subjects. Due to its technical properties, the C-Quant is a useful device when evaluating the long-term effect of surgical procedures on the quality of vision.
Fig. 2 Shows the effect of age on straylight along with the fitted linear regression line

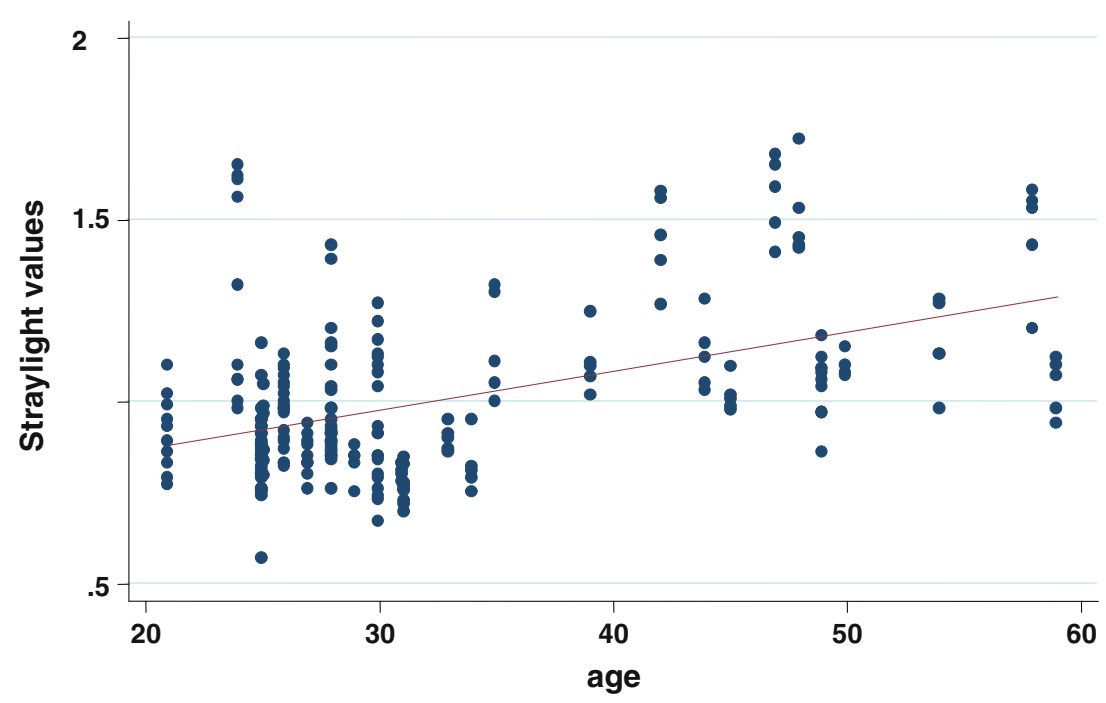


Conflict of interest None.

\section{References}

1. van Rijn LJ, Wilhelm H, Emesz M, Kaper R, Heine S, Nitsch S, Grabner G, Volker-Dieben HJ (2002) Relation between perceived driving disability and scores of vision screening tests. $\mathrm{Br} \mathrm{J}$ Ophthalmol 86:1262-1264

2. Elliott DB, Bullimore MA (1993) Assessing the reliability, discriminative ability, and validity of disability glare tests. Invest Ophthalmol Vis Sci 34:108-119

3. van den Berg TJ (1986) Importance of pathological intraocular light scatter for visual disability. Doc Ophthalmol 61:327-333

4. Vos JJ (1984) Disability glare-a state of the art report. Comm Int Eclairage J 3:39-53

5. Beckman C, Hard S, Hard AL, Sjostrand J (1992) Comparison of two glare measurement methods through light scattering modeling. Optom Vis Sci 69:532-537

6. Coppens JE, Franssen L, van den Berg TJ (2006) Reliability of the compensation comparison method for measuring retinal stray light studied using Monte-Carlo simulations. J Biomed Opt 11:054010

7. Franssen L, Coppens JE, van den Berg TJ (2006) Compensation comparison method for assessment of retinal straylight. Invest Ophthalmol Vis Sci 47:768-776
8. Cervino A, Montes-Mico R, Hosking SL (2008) Performance of the compensation comparison method for retinal straylight measurement: effect of patient's age on repeatability. $\mathrm{Br} \mathrm{J}$ Ophthalmol 92:788-791

9. van Rijn LJ, Nischler C, Gamer D, Franssen L, de Wit G, Kaper R, Vonhoff D, Grabner G, Wilhelm H, Volker-Dieben HJ, van den Berg TJ (2005) Measurement of stray light and glare: comparison of Nyktotest, Mesotest, stray light meter, and computer implemented stray light meter. Br J Ophthalmol 89:345-351

10. Landis JR, Koch GG (1977) The measurement of observer agreement for categorical data. Biometrics 33:159-174

11. Rozema JJ, Van den Berg TJ, Tassignon MJ (2010) Retinal straylight as a function of age and ocular biometry in healthy eyes. Invest Ophthalmol Vis Sci 51:2795-2799

12. Koch DD (1989) Glare and contrast sensitivity testing in cataract patients. J Cataract Refract Surg 15:158-164

13. van den Berg TJ (1991) On the relation between glare and straylight. Doc Ophthalmol 78:177-181

14. Westheimer G, Liang J (1995) Influence of ocular light scatter on the eye's optical performance. J Opt Soc Am A Opt Image Sci Vis 12:1417-1424

15. Rubin GS, Roche KB, Prasada-Rao P, Fried LP (1994) Visual impairment and disability in older adults. Optom Vis Sci 71:750760

16. Rubin GS, Stark WJ (1995) Current status of contrast sensitivity and glare testing in evaluation of the cataract patient. Ophthalmol Clin North Am 8:539-546 